\title{
The Quality Control of Alcoholic Components of Disinfectants by a Simple Colour Test
}

\author{
Heinz Langhals* (iD $\$$ \\ LMU University of Munich, Department of Chemistry, Butenandtstr. 13, D-81377 Munich, Germany. \\ Received 5 May 2020, revised 26 June 2020, accepted 26 June 2020.
}

\begin{abstract}
The identity and quality of liquid components for disinfectants, preferentially isopropyl alcohol and ethanol, can be determined with the naked eye using solvatochromic dyes and comparing their colour with a colour scale. Thus, any confusion with toxic methanol or other solvents can be excluded, as can also the application of raw materials in insufficient concentration or incorrect formulations. The production of a quick and easy to use simple low-cost test kit is described.
\end{abstract}

KEYWORDS

Disinfectants, alcohols, analytics, colour test, quality control.

\section{Introduction}

Disinfectants are one of the most efficient tools for the control of contact diseases and is becoming more important in pandemic spreads such as is currently the case with SARS-CoV-2. Disinfections of the hands with disinfectants may be achieved using comparably small amounts, requiring less water than detergents, such as soaps, and being generally more effective, is of central importance; however, disinfectants allow the application of only a very limited variety of components, because skin irritations are caused by frequent use. Thus the World Health Organisation (WHO) recommends isopropyl alcohol (IPA; CAS registry number RN 67-63-0) between $60 \%$ (activity decreases rapidly below $50 \%$ ) and $90 \%$ concentrations, where $75 \%$ is used for the safe Formulation 2; some hydrogen peroxide is added as a booster and some glycerol for skin protection. ${ }^{1}$ If there is no isopropyl alcohol available, Formulation 1 containing ethanol (RN 64-17-5) in a higher concentration of $80 \%$ is recommended as an alternative. Generally, the pharmaceutical industry provides certified isopropyl alcohol; however, the widespread supply may become problematic in pandemic situations. Therefore, it may become necessary to procure the components for disinfectant from various other technical sources, such as directly from suppliers in the chemical industry where local production of disinfectants would be helpful. Chemical analysis would be necessary in such cases, with the focus on confirming the safety of the chosen alcohol, determining the water content for sufficient activity, and verifying the alcohol content of the prepared formulation. A simple test, which could be carried out by less skilled or educated persons would be helpful, while a visual colour test without analytical equipment would be most effective.

\section{Materials and Methods}

The primary dye used in this study, namely $\mathbf{1}$ (phenolate betaine) $\left(E_{\mathrm{T}}(30), \mathrm{RN}\right.$ 10081-39-7) developed by Dimroth and Reichardt, ${ }^{2}$ is commercially available. The handling of $\mathbf{1}$ proved to be unproblematic and no adverse effects of $\mathbf{1}$, such as toxicity, are known according to the Chemical Abstracts applied with the SciFinder ${ }^{\circledR}$. Only very low concentrations, such as dyes in inks, are applied; however, $\mathbf{1}$ should be handled with care like other

* To whom correspondence should be addressed.

E-mail: langhals@Irz.uni-muenchen.de

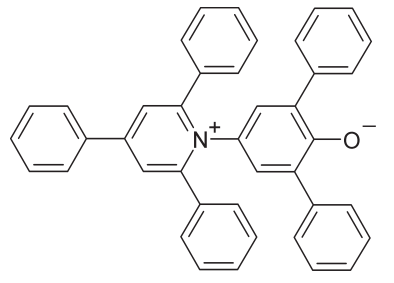

1

only partially tested chemicals; only very low quantities are required for test kits, where the price of the dye used in the test kit is of minor importance. The dye can be synthesized according to the literature. $2,3,4$

A stock solution of $\mathbf{1}$ is prepared in acetone with a maximal concentration according to the colouration in Fig. 1B; the colour is deeper in larger vessels because of the larger optical path lengths). Acetone is recommended because of its general availability, low toxicity and easy evaporation. $10 \mathrm{~mL}$ samples of the green stock solution were transferred into small sample glass vials of $20 \mathrm{~mL}$ total volume with a white, colourless screw cap (the latter does not interfere with the perception of colour). The filling height (level) was marked (Fig. 1B) and the acetone was allowed to evaporate in a fire-protected, ventilated environment with the exclusion of direct light (because of the limited lightfastness of 1) until dry at room temperature, or heated to below the boiling point of acetone $\left(56^{\circ} \mathrm{C}\right)$. The caps of the dye-doped sample glass vials (Fig. 1C) were screwed on for sealing to obtain the test kit ready for use.

The alcohol for testing is filled into dye-doped sample glass vials up to the marked level (Fig. 1D) and the colour hue of the dissolved dye is compared with a colour scale (Tables 1 and 2).

The colour coordinates in the RGB room were directly taken

Table 1 Colour coordinates of the solution of $\mathbf{1}$ in various solvents.

\begin{tabular}{llrrr}
\hline \multirow{2}{*}{ Substance } & Colour & \multicolumn{3}{c}{ Colour coordinates RGB } \\
\cline { 3 - 5 } & & $\mathrm{R}$ & $\mathrm{G}$ & $\mathrm{B}$ \\
\hline \multirow{2}{*}{ Isopropyl alcohol } & Blue & 37 & 72 & 136 \\
Ethanol & Violet & 112 & 76 & 122 \\
Methanol & Red & 149 & 103 & 114 \\
Acetone & Green & 27 & 89 & 84 \\
\hline
\end{tabular}




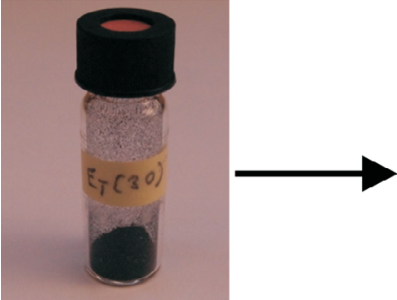

A

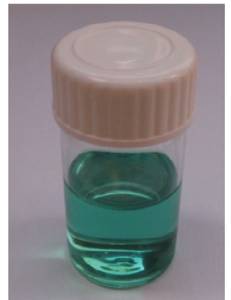

B

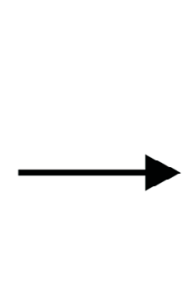

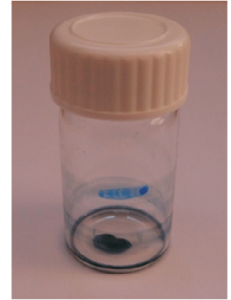

$\mathrm{C}$

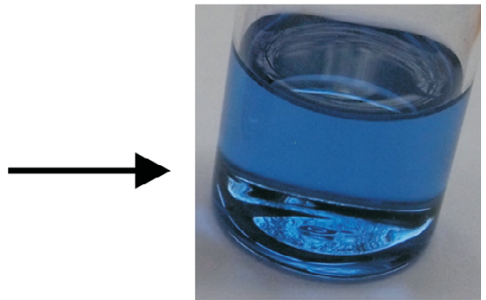

$\mathrm{D}$

Figure 1 A simple test kit for the identification of alcohols and the determination of the water content. A: Starting with solid crystalline 1 and preparation of a green stock solution in acetone; B: Filling small sample glass vials and marking the levels, C: Evaporation of the acetone until dryness; the test kit is prepared and ready to use or to be transported/sent; D: Filling the alcohol to be tested up to the marked level, dissolution of the dye and comparing the colour with a colour scale.

Table 2 Colour coordinates of solutions of $\mathbf{1}$ in various alcohol-watermixtures.

\begin{tabular}{|c|c|c|c|c|c|c|}
\hline \multirow{3}{*}{$\begin{array}{c}\text { Alcohol content } \\
\qquad / \%\end{array}$} & \multicolumn{6}{|c|}{ Colour coordinates RGB } \\
\hline & \multicolumn{3}{|c|}{ Isopropyl alcohol } & \multicolumn{3}{|c|}{ Ethanol } \\
\hline & $\mathrm{R}$ & G & B & $\mathrm{R}$ & $\mathrm{G}$ & B \\
\hline 100 & 32 & 66 & 129 & 85 & 45 & 98 \\
\hline 95 & 58 & 67 & 126 & 100 & 50 & 99 \\
\hline 90 & 87 & 77 & 130 & 122 & 74 & 114 \\
\hline 85 & 104 & 80 & 128 & 133 & 88 & 117 \\
\hline 80 & 118 & 92 & 127 & 129 & 94 & 114 \\
\hline 75 & 131 & 104 & 123 & 133 & 104 & 122 \\
\hline 70 & 136 & 113 & 129 & 135 & 109 & 120 \\
\hline 65 & 139 & 119 & 128 & & & \\
\hline 60 & 134 & 117 & 125 & & & \\
\hline
\end{tabular}

from a digital photograph shot in daylight (camera Olympus EP-1 with colour correction for the overcast sky and the software PHOTOED.EXE) and are comparatively precise despite the unfavourable optic of the round glass vessels. The coordinates are given for the pure solvents in Table 1 and the alcohol-water mixtures in Table 2, allowing a digital reproduction of the colours; thus, distilled water was placed in $0.5 \mathrm{~mL}$ steps and each filled up to a total volume of $10 \mathrm{~mL}$, for example, $1.5 \mathrm{~mL}$ of water was filled up to a total volume of $10 \mathrm{~mL}$ to give $85 \%$ alcohol. Volume contractions caused by mixing were unimportant for the colour effect and could be neglected; however, they can be calculated. ${ }^{5}$ The coordinates allow the printing of colour scales; however, the reproduction of colour on the screens of modern flat screens of various manufactures for computers or smartphones (Samsung Galaxy S8 was applied here) is precise enough for a direct colour comparison. The screens should operate without colour correction or set to purely white; the setting of parameters is of minor importance.

Dye 1 may be recovered from the used solution.

There are alternatives if the availability of $\mathbf{1}$ is limited. The solvatochromism of the more easily available dye $2\left(E_{\mathrm{T}}(33), \mathrm{RN}\right.$ 121792-58-3 $)^{4}$, nearly equals the effect of $\mathbf{1}$ and an appreciable solvatochromism is still given by dye 3 (MOED, RN 23302-83-2), ${ }^{6}$ where the latter can be prepared in laboratory batches of more than $100 \mathrm{~g}$; however, specific colour scales have to be established for $\mathbf{2}$ and 3, because of different scopes of solvatochromism.

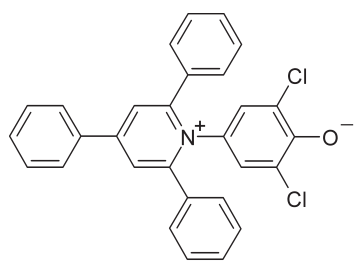

2

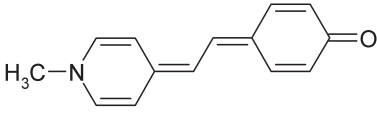

\section{Results and Discussion}

We used the solvatochromic dye $\mathbf{1}\left(E_{\mathrm{T}}(30), \mathrm{RN} 10081-39-7\right)$ as a test reference for mediums, because the light absorption of solutions is characteristic of the applied pure solvent, where the effect is dominated by the solvent polarity. Small contents of water are indicated by hypsochromic shifts, because of the very high polarity of the latter (the effect is interpreted in terms of more efficient solvation of the polar ground state of $\mathbf{1}$ compared with the less polar first electronically excited state; for more general background information see ref.7); the effect is so strong that alcohols can be visually identified using the dissolution of $\mathbf{1}$ (Fig. 2).

Characteristic blue solutions of $\mathbf{1}$ are obtained in isopropyl alcohol, whereas solutions in ethanol are violet; see Fig. 2. This allows the identification of the alcohols, namely isopropyl alcohol and ethanol, by a simple comparison of the colour hue with a colour scale, such as in Table 1 . Moreover, a clear differentiation from the toxic methanol is possible, because the latter forms red solutions. Even an erroneous confusion with the water-mixable acetone would become obvious, because of the green colour of the solution of $\mathbf{1}$ (see Fig. 1B). Strong deviations in colour would also be found for other solvents.

The concentration of alcohols may be routinely determined based on their density using areometers. Also, a method for the determination of water in organic solvents was developed for a spectrometer. ${ }^{8}$ However, this requires special equipment and skills and maybe problematic in pandemic situations. Whereas a simple colour test would require no additional equipment and experience.

As mentioned previously, the colour hue of $\mathbf{1}$ is strongly influenced by the water content of a medium, because of the high polarity of water. For this reason, this method can be simplified to a visual comparison of a colour hue (as shown in Fig. 3), where changes in colour are clearly visible for $5 \%$ steps in the concentration of alcohol. This is still sensitive enough for a test of the quality of the materials for disinfectants.

The local availability of isopropyl alcohol may be limited in pandemic situations, whereas ethanol is more generally accessible not only by industrial chemical synthesis but also by alco-

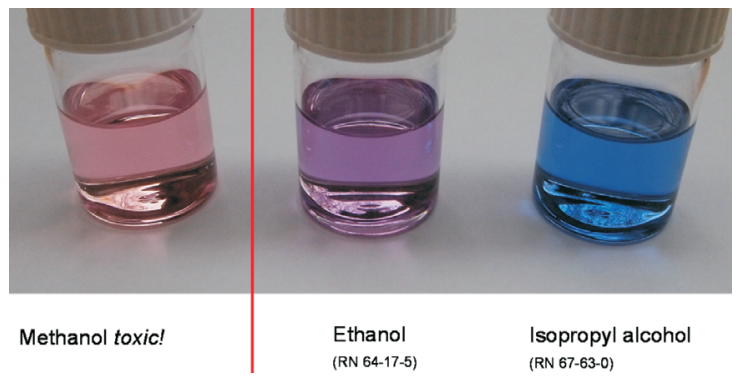

Figure 2 Colour hues of solutions of $\mathbf{1}$ in methanol, ethanol (for Formulation 1) and isopropyl alcohol (for Formulation 2). 


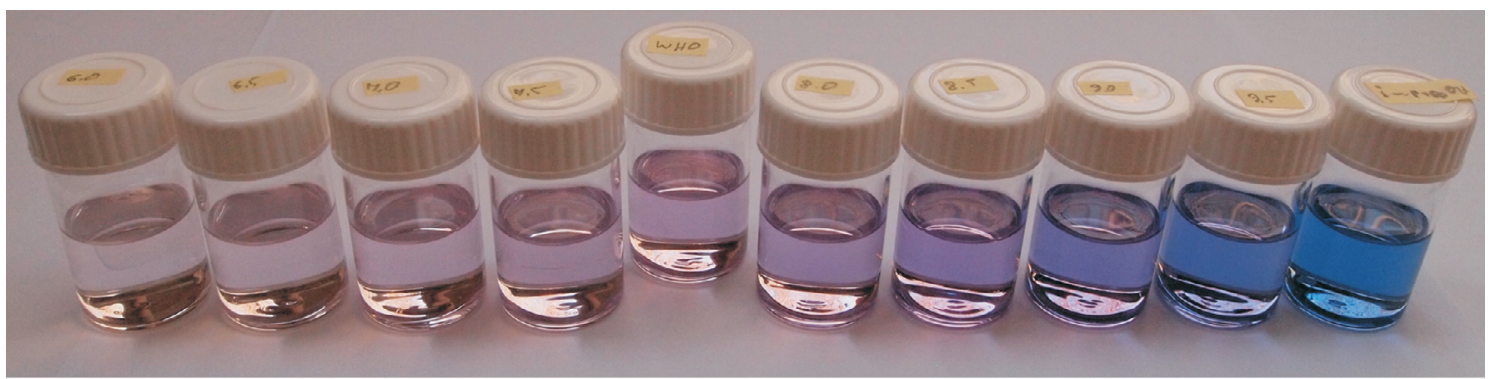

\section{$60 \% \quad 65 \% \quad 70 \% \quad 75 \% \quad$ WHO2 $\quad 80 \% \quad 85 \% \quad 90 \% \quad 95 \% \quad 100 \%$}

Figure 3 Colour scale of 1 for water containing isopropyl alcohol (RN 67-63-0) with the percentage of the alcohol shown below each sample. (WHO2: Formulation 2 recommended by the WHO.)

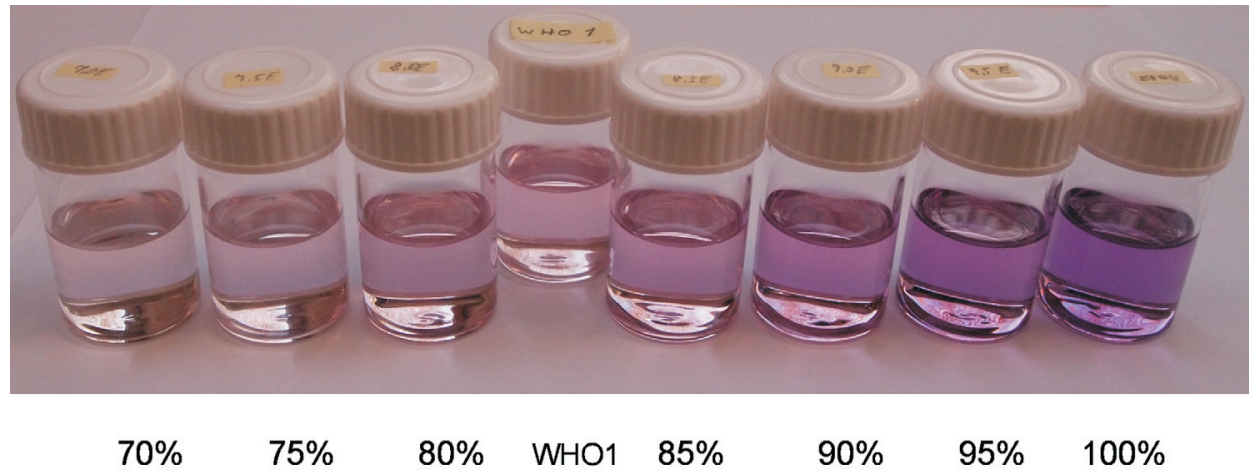

Figure 4 Colour scale of 1 for water-containing ethanol (RN 64-17-5) with the percentage of alcohol shown below each sample. (WHO1: Formulation 1 recommended by the WHO.)

holic fermentation. Therefore, Formulation 1 of WHO based on ethanol is an interesting alternative. However, the therapeutic index is smaller and higher concentrated $(80 \%)$ ethanol must be used. The water content of ethanol can be determined in the same way as for the isopropyl alcohol, where the colour scale is indicated in Fig. 4. The colour shift caused by water is not as pronounced as for isopropyl alcohol starting with the violet colour for the latter, because of the higher polarity of ethanol; however, changes are strong enough for an unequivocal determination of the water content; see Fig. 4.

The solvatochromic dye $\mathbf{1}$ is strongly light absorbing, where the molar absorptivity of the solvatochromic band of 5500 in isopropyl alcohol and of 4400 in ethanol is described; 2 it slightly decreases with the addition of water. The high absorptivity is advantageous, because $\mathrm{mg}$ amounts per $10 \mathrm{~mL}$ are required for visible colourations; however, individual dosing by weight requires an ultra-microbalance instrument.

On the other hand, sufficiently exact dosing without using additional equipment can succeed by comparing the colouration using a test kit prepared as shown in Fig. 1 with the colour scales (Tables 1 and 2).

\section{Conclusion}

A simple, quick and safe colour test for the identification of isopropyl alcohol and ethanol as components for disinfectants has been developed. This colour test proceeds by comparing the colour hue of dissolved phenolate betaine 1 with a colour scale. Additionally, the water content of such materials can be effectively determined in the same way as the proper content controlled in the final disinfectant. The preparation of a very simple low-cost test kit suitable for the use by low-skilled persons and containing a dosed amount of $\mathbf{1}$ is also described.

\section{${ }^{5}$ ORCID ID}

H. Langhals: (iD orcid.org/0000-0002-8038-4547

\section{References}

1 https://www.who.int/gpsc/5may/Guide_to_Local_Production.pdf; 5 May 2020.

2 K. Dimroth, C. Reichardt, T. Siepmann and F. Bohlmann, About pyridinium- $N$-phenolate betaines and their application for the characterisation of the polarity of solvents, Justus Liebigs Ann. Chem., 1963, 661, 1-37.

$3 \mathrm{H}$. Langhals, The quantitative description of the solvent polarity of binary mixtures considering various polarity scales, Chem. Ber., 1981, 114, 2907-2913.

4 M.A. Kessler and O.S. Wolfbeis, An improved synthesis of the solvatochromic dye ET-30, Synthesis, 1988, 635-636.

5 H. Langhals, Simplification of the analysis of binary liquid mixtures by considering the index of refraction and the density, Zeitschr. Analyt. Chem., 1984, 319, 293-295.

6 L.G.S. Brooker, G.H. Keyes and D.W. Heseltine, Color and constitution. XI. Anhydronium bases of $p$-hydroxystyryl dyes as solvent polarity indicators, J. Am. Chem. Soc., 1951, 73, 5350-5256.

7 H. Langhals, Heterocyclic structures applied as efficient molecular probes for the investigation of chemically important interactions in the liquid phase, Chem. Heterocycl. Compd., 2017, 53, 2-10. DOI: 10.1007/s10593-017-2014-z

8 H. Langhals, A simple, quick, and precise procedure for the determination of water in organic solvents, Anal. Lett., 1990, 23, 2243-2258. 